\title{
Photometry of R Coronae Borealis Stars during the Recovery Phases of Their Declines
}

\author{
Ljiljana Skuljan, Peter L. Cottrell \\ Department of Physics and Astronomy, University of Canterbury, \\ Private Bag 4800, Christchurch 8020, New Zealand
}

\begin{abstract}
R}$ Coronae Borealis ( $\mathrm{RCB}$ ) stars are a rare class of variable stars, well-known by their declines, but still posing two fundamental questions: what is the origin and what is the nature of their unpredictable minima? Possible contributions to the answering of these questions could use large-scale surveys, by discovering new RCB stars. In addition, photometric databases from these surveys and other long-term photometric programs of targeted stars can be used to compile information about these decline phases.

The photometric observations ( $U B V R I$ ) of nine cool RCB stars have been collected at Mt John University Observatory, New Zealand, for the last ten years. Analysis of the magnitude-color diagrams for the recovery phase of a number of declines of these stars has been made in order to determine the extinction properties of the dust. The photometric magnitudes and colors show significant changes during the RCB declines. The asymptotic approach to the normal brightness can be seen on the magnitude-color diagrams from the recovery phase. This is observed for all RCB declines, and without exception is independent of their decline amplitude. According to the most acceptable theory, the distribution of the dust matter in the cloud which causes the light fading can be considered uniform as the stars recover to the maximum brightness. The slopes of the magnitude-color and color-color diagrams from the recovery phase of 26 observed $\mathrm{RCB}$ declines have been used in order to define the normalized extinction quantity $A_{\lambda} / A_{V}$. Although the extinction curve in the $U B V R I$ region is not a good indicator of the type and size of the particles, our data show that the material causing the RCB declines has extinction properties similar to that for the interstellar medium. The reddening parameter $\left(R_{B V}=A_{V} / E(B-V)\right)$ for the $\mathrm{RCB}$ stars in our sample is in the range 2.5 to 4.6 , similar to the interstellar dust. Observations have been compared with the theoretical extinction curves for different sorts of grains. The extinction properties in the $U$ region follow the trend of the UV data from other RCB observations as well as laboratory data for amorphous carbon.
\end{abstract}

